\title{
Article \\ Biochar and Fertilization Effects on Weed Incidence in Winter Wheat
}

\author{
Bojana Brozović *, Irena Jug DD, Danijel Jug, Bojan Stipešević, Marija Ravlić and Boris Đurđević (D) \\ Faculty of Agrobiotechnical Sciences Osijek, Josip Juraj Strossmayer University of Osijek, Vladimira Preloga 1, \\ HR-31000 Osijek, Croatia; ijug@fazos.hr (I.J.); danijel.jug@fazos.hr (D.J.); bojan.stipesevic@fazos.hr (B.S.); \\ marija.ravlic@fazos.hr (M.R.); boris.djurdjevic@fazos.hr (B.Đ.) \\ * Correspondence: bojana.brozovic@fazos.hr; Tel.: +385-31-554-896
}

Citation: Brozović, B.; Jug, I.; Jug, D.; Stipešević, B.; Ravlić, M.; Đurđević, B. Biochar and Fertilization Effects on Weed Incidence in Winter Wheat. Agronomy 2021, 11, 2028. https:// doi.org/10.3390/agronomy11102028

Academic Editor: Anestis Karkanis

Received: 30 August 2021

Accepted: 6 October 2021

Published: 9 October 2021

Publisher's Note: MDPI stays neutral with regard to jurisdictional claims in published maps and institutional affiliations.

Copyright: (c) 2021 by the authors. Licensee MDPI, Basel, Switzerland. This article is an open access article distributed under the terms and conditions of the Creative Commons Attribution (CC BY) license (https:// creativecommons.org/licenses/by/ $4.0 /)$

\begin{abstract}
Biochar, a carbon-rich material, is highlighted to improve soil fertility, simultaneously mitigating climate change by carbon sequestration. Combined with mineral fertilizer, it can increase weediness, the major source of yield loss in agricultural production. Research with biochar was conducted in Eastern Croatia in 2016, with the aim to investigate the influence of biochar and mineral fertilizer on weed infestation and winter wheat yield. Field experiments were set up as a split-plot where biochar (B) was the main factor and fertilization was the sub factor. The main treatments were: B0 (control without biochar), B1-5 $\mathrm{t} \mathrm{ha}^{-1}$, B2-10 $\mathrm{t} \mathrm{ha}^{-1}$ and B3-15 $\mathrm{t} \mathrm{ha}^{-1}$. Fertilization sublevels were F0) without fertilizer and F1) optimal dose of fertilizer. Weediness was determined by counting and measuring aboveground biomass. The treatments with the greatest effect on weediness were B3 and F1 in the winter wheat tillering stage. In the winter wheat ripening stage, treatment B3 obtained the highest weediness and F1 significantly reduced weed density. Biochar treatment B3 increased winter wheat yield by $77 \%$ in relation to the control. The application of biochar combined with fertilization affected the level of weediness depending on agroecological conditions, but with a significant increase in yield.
\end{abstract}

Keywords: black carbon; mineral fertilizer; weediness; Triticum aestivum L.; yield

\section{Introduction}

Agricultural production is currently at the center of the challenge of adaptation to climate change. In recent years, climate change has affected the precipitation and temperature regime with the increasing occurrence of extreme weather conditions (e.g., drought, high temperatures) [1,2]. Such conditions affect crop productivity, including that of winter wheat, which is the most important food source today [3]. A way to mitigate adverse weather events is to maintain or improve soil fertility, and one of the solutions is the use of biochar. Biochar is a carbon-rich product of pyrolysis which is seen as a significant tool in climate change mitigation due to carbon sequestration in the soil and a reduction in greenhouse gas emissions [4-6]. Biochar produced from various organic substances is more often used in agricultural production because it serves as a soil amended with the aim of improving soil fertility and crop productivity [7]. Biochar has the potential to increase crop productivity in different plant production systems through a wide range of effects at different soil functions. The physical, chemical and biological properties of the soil show a positive response to biochar application $[4,8,9]$. Increasing crop productivity is associated with an improvement in nutrient retention and nutrient cycling, better water holding capacity, bulk density reduction and increased microbiological activity and organic matter formation $[10,11]$. Biochar $\mathrm{pH}$ value is commonly neutral or basic and therefore has a liming effect, with a positive impact on acidic and neutral soils $[5,9,12]$. In addition to the current knowledge on increasing crop productivity by adding biochar, it is realistic to expect that this effect will be reflected in weeds, which are indispensable companions of the plant production system. It is assumed that the addition of biochar 
will increase weediness and competition with the crops [13]. Although weeds are a major factor that negatively affects the productivity of agricultural production and can account for almost $50 \%$ of the loss of possible wheat yield, there is little research dealing with the impact of biochar on weeds, especially in field conditions [14-18]. According to previous research, it was proven that biochar reduces the need for fertilization by keeping yields stable $[7,19]$. Optimal fertilization can benefit weeds more than crops because weeds have an advantage in nutrient uptake over crops and are strongly influenced by nutrient sources and available nutrients [20-22]. However, optimal fertilization can also lead to a reduction in weediness by increasing the benefits of the crop as a competitor and can be an important part of integrated weed management $[15,23,24]$. The question arises as to how the mutual application of biochar and fertilizer affects the occurrence of weeds, and, ultimately, the yield of the crop. The synergistic effect of biochar and fertilization has been proven in terms of increasing crop productivity compared to the individual application of biochar or fertilizer [5]. According to a few previous studies, the simultaneous application of biochar and mineral fertilizer resulted in a yield increase, while the increase in yield was absent with the application of biochar alone $[25,26]$. The application of biochar and nitrogen fertilizer did not significantly affect the abundance of weeds in maize, while the application of biochar alone led to an increase in the number of weeds in wheat, and weed density also depended on the different applied doses of biochar $[27,28]$. Additionally, an increase in wheat yield during drought conditions with biochar application was reported [29]. The potential of biochar to increase weediness was also proven in research where, in combination with fertilizer, it caused an increase in weed cover [17]. However, the negative impact of biochar on weed occurrence was proven by a study where a reduction in weed emergence after biochar incorporation was recorded [18]. Because of insufficient and contradictory previous results on the impact of biochar on weeds in field conditions, there is a need for further research. In this research, biochar was used for the first time as a soil conditioner on acid soils in Croatia. The hypothesis of this research was that biochar alone and in combination with NPK fertilizer would increase winter wheat yield and reduce weed pressure. The aim of the study was therefore to investigate the influence of biochar in different amounts, alone or in combination with optimal NPK fertilization, on weediness and winter wheat yield.

\section{Materials and Methods}

\subsection{Experimental Site and Design}

The research with biochar as a soil conditioner was conducted in the eastern region of Croatia, in 2016. The field experiments were conducted in Osijek-Baranja County (Lon. 17.3327 E Lat. 45.6975 N Alt. $89.00 \mathrm{~m}$ - site Gat) and Virovitica-Podravina County (Lon. 17.8587 E, Lat. $45.61125 \mathrm{~N}$ Alt. $107.00 \mathrm{~m}$-site Cacinci) on acid soils (Table 1) in mainly lowland area with some hilly parts in the western part of the region [30].

Table 1. Description of the basic properties of soil types on two experimental field sites.

\begin{tabular}{|c|c|c|}
\hline & Gat (G) & Cacinci (C) \\
\hline Soil type/soil texture & Gleysol/SL & Stagnosol/SLC \\
\hline $\mathrm{pH} \mathrm{H}_{2} \mathrm{O} / \mathrm{KCl}$ & $5.12 / 4.00$ & $5.00 / 3.75$ \\
\hline SOM (\%) & 1.40 & 2.32 \\
\hline Hy $\left(\mathrm{cmol} \mathrm{kg}^{-1}\right)$ & 5.80 & 9.12 \\
\hline $\mathrm{AL}-\mathrm{P}_{2} \mathrm{O}_{5}\left(\mathrm{mg} 100 \mathrm{~g}^{-1}\right)$ & 6.20 & 8.10 \\
\hline $\mathrm{AL}-\mathrm{K}_{2} \mathrm{O}\left(\mathrm{mg} 100 \mathrm{~g}^{-1}\right)$ & 16.20 & 14.20 \\
\hline Sand, silt, clay $\%(0-36 \mathrm{~cm})$ & $22.69,59.30,18.01$ & $14.38,55.46,30.16$ \\
\hline
\end{tabular}


The soil types were determined according to IUSS Working Group WRB [31]. The research area is characterised by a mean annual temperature from 9 to $11^{\circ} \mathrm{C}$, with an increasing trend from west to east and from northwest to northeast.

The average amount of precipitation ranges from $600 \mathrm{~mm}$ in the eastern to $800 \mathrm{~mm}$ in the western part of the region (Meteorological and Hydrological Institute of Croatia). The experiment was set as a split-plot design in four repetitions at both locations with a basic plot area of $8 \mathrm{~m} \times 10 \mathrm{~m}$, with winter wheat used as a crop. The biochar (B) treatment was used as a main factor. Biochar treatments were: B0 (control without biochar), B1-5 $\mathrm{t} \mathrm{ha}^{-1}$, B2-10 $\mathrm{t} \mathrm{ha}^{-1}$ and B3-15 $\mathrm{t} \mathrm{ha}^{-1}$. Each basic plot was divided into two fertilization sublevels, F1) optimal dose of mineral fertilizer and F0) without mineral fertilizer, as sub factors (Table 2). The optimal dose of mineral fertilizer was calculated using the ALRxp computer program for fertilizer recommendations [32].

Table 2. Fertilization according to recommendation.

\begin{tabular}{|c|c|c|c|}
\hline Location & $\mathrm{P}_{2} \mathrm{O}_{5}\left(\mathrm{~kg} \mathrm{ha}^{-1}\right)$ & $\mathrm{K}_{2} \mathrm{O}\left(\mathrm{kg} \mathrm{ha}^{-1}\right)$ & $\mathrm{N}\left(\mathrm{kg} \mathrm{ha}^{-1}\right)$ \\
\hline Gat & 150 & 42 & 168 \\
\hline Cacinci & 150 & 47 & 158 \\
\hline
\end{tabular}

\subsection{Weather Conditions during Investigations at Two Experimental Sites}

The data of average temperatures and precipitations during the research experiment at both locations are shown in Figures 1 and 2 (Source: Croatian Meteorological and Hydrological Service, 2017). In the investigated period, at the Cacinci location, less precipitation was recorded $(595 \mathrm{~mm})$ compared to the long-term average (1981/2015), which is 728.5 . The exception was July, when more than $100 \mathrm{~mm}$ of precipation was recorded. In the period from March to June, a lack of precipitation was present compared to the long-term average.

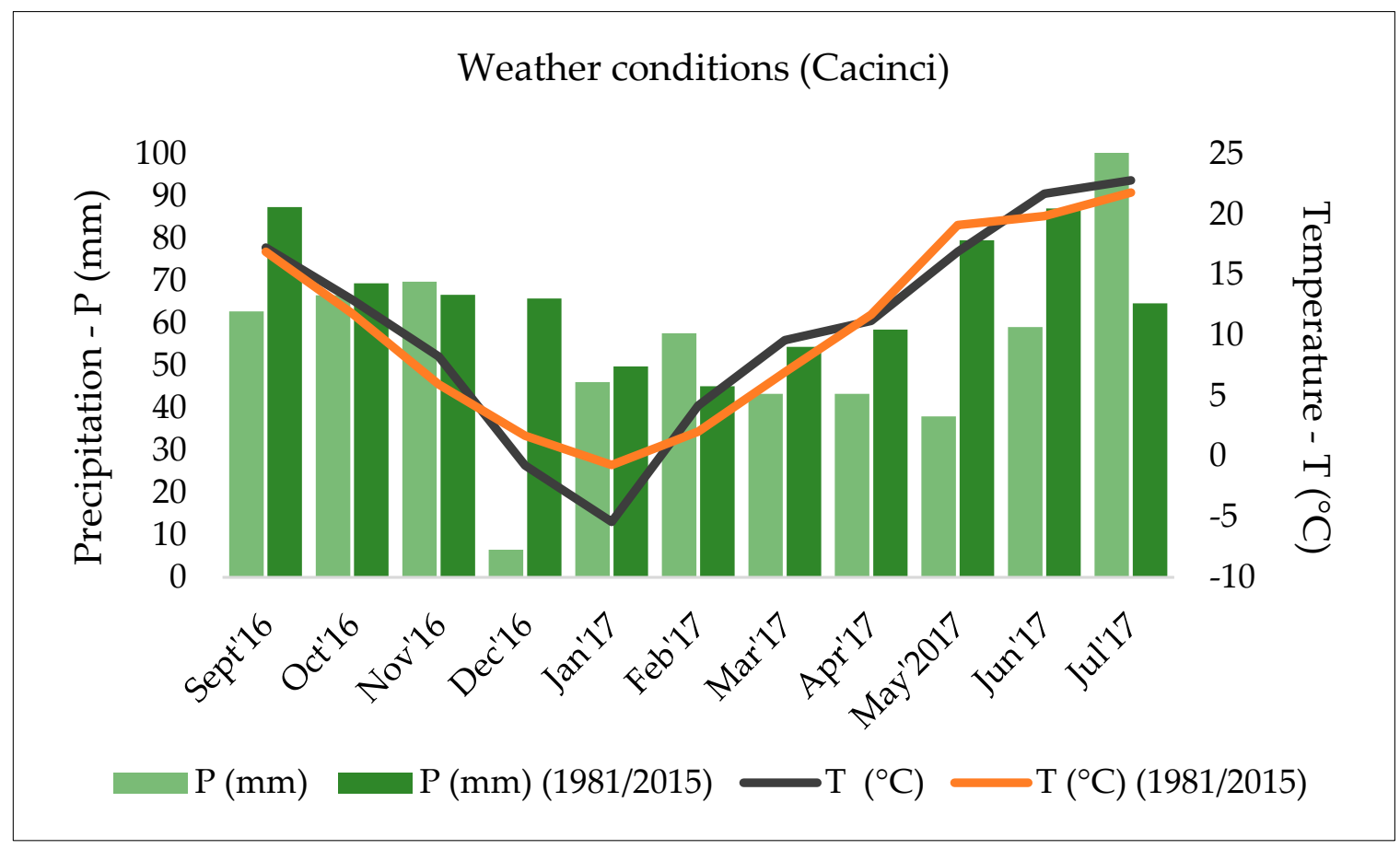

Figure 1. Average temperature and precipitation in winter wheat vegetation (September 2016-July 2017) and long-term average (1981/2015) for the Cacinci experimental site. 


\section{Weather conditions (GAT)}

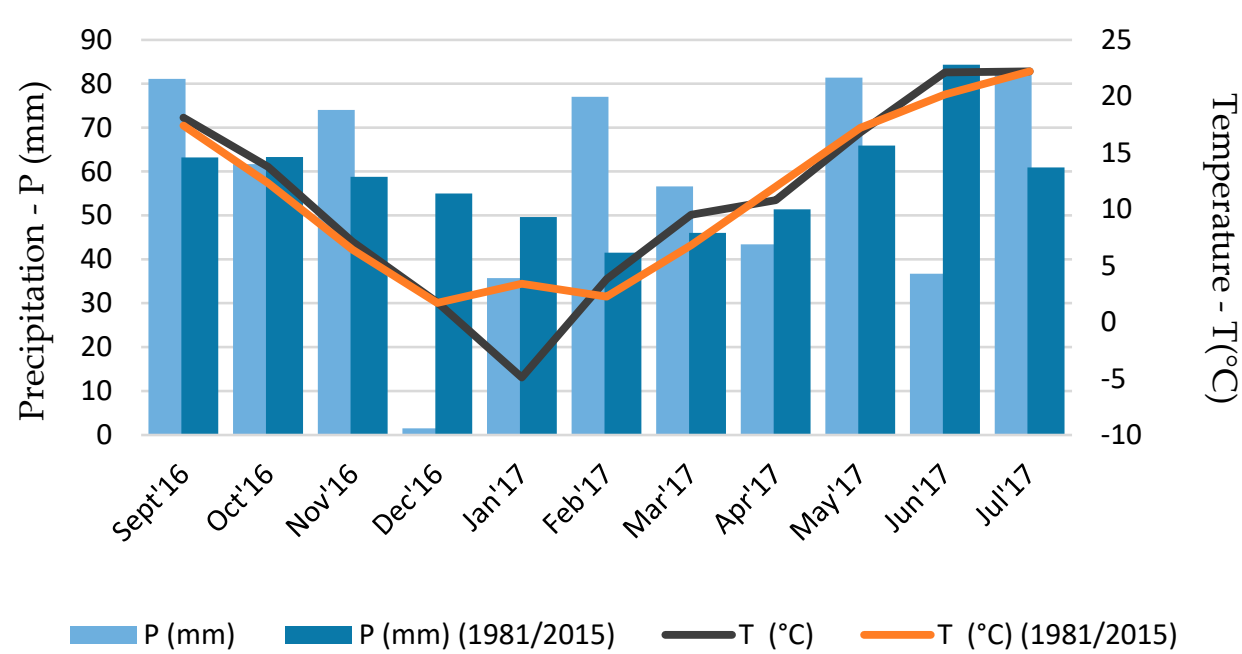

Figure 2. Average temperature and precipitation in winter wheat vegetation (September 2016-July 2017) and long-term average (1981/2015) for the Gat experimental site.

The precipitation deficit was almost $100 \mathrm{~mm}$ compared to the long-term average. Average temperatures in winter wheat vegetation did not deviate significantly from the long-term average. Looking at the average amount of precipitation for the investigated period at the Gat site $(632 \mathrm{~mm})$, values did not differ significantly from the long-term average (1981/2015-640 mm) (Figure 2). However, noticeably higher amounts of precipitation were recorded for some months in the winter wheat vegetation. Almost $50 \%$ more precipitation than the long-term average fell in February. A higher amount of precipitation was recorded in May and July $(82$ and $83 \mathrm{~mm}$ ) compared to the long-term average. A pronounced lack of precipitation was present at both experimental sites in December. Only $1.5 \mathrm{~mm}$ of precipitation was recorded at the Gat site (long-term average: $55 \mathrm{~mm}$ ) while 6.5 $\mathrm{mm}$ was recorded at the Cacinci site (long-term average: $66 \mathrm{~mm}$ ). At both experimental locations, the amount of precipitation was unevenly distributed during the winter wheat vegetation. Average temperatures during winter wheat vegetation at the Gat site did not differ significantly from the long-term average. The exception was the month of January, when significantly lower temperatures were measured at both locations.

\subsection{Field Methods}

The biochar was incorporated into the soil to a depth of $25 \mathrm{~cm}$ (chiseling) six months before maize seeding (previous crop to winter wheat) due to the required incubation in soil. The particle size of the produced biochar ranged from 1 to $10 \mathrm{~mm}$. In this experiment, the biochar was produced from waste wood that was collected in oak and beech blend woods near the experimental sites. The process of slow pyrolysis was executed at $400{ }^{\circ} \mathrm{C}$ under limited oxygen conditions and lasted for $24 \mathrm{~h}$. The chemical characterization of biochar is given in Table 3.

Table 3. Chemical characterization of biochar.

\begin{tabular}{|c|c|c|c|c|c|c|c|c|c|}
\hline \multicolumn{10}{|c|}{ Total Amounts of Microelements and Heavy Metals (mg kg ${ }^{-1}$ ) } \\
\hline As & $\mathrm{Cd}$ & Co & $\mathrm{Cr}$ & $\mathrm{Cu}$ & $\mathrm{Fe}$ & $\mathrm{Mn}$ & $\mathrm{Ni}$ & $\mathrm{Pb}$ & $\mathrm{Zn}$ \\
\hline 1.7 & 0.1 & 1.0 & 2.7 & 8.3 & 2856.0 & 358.0 & 4.4 & 2.2 & 12.0 \\
\hline \multicolumn{10}{|c|}{ Total Amounts of Macroelements (\%) } \\
\hline \multicolumn{2}{|c|}{ C } & \multicolumn{2}{|c|}{$\mathrm{Ca}$} & \multicolumn{2}{|c|}{$\mathrm{K}$} & \multicolumn{2}{|c|}{$\mathrm{Mg}$} & \multicolumn{2}{|c|}{$\mathrm{P}$} \\
\hline \multicolumn{2}{|c|}{70.13} & \multicolumn{2}{|c|}{1.9} & \multicolumn{2}{|c|}{0.28} & \multicolumn{2}{|c|}{0.12} & \multicolumn{2}{|c|}{0.04} \\
\hline
\end{tabular}


Mineral fertilization and basic soil tillage (chiseling) were carried out on 19 October at site Gat and 23 October at site Cacinci. The total amount of $\mathrm{P}$ and $\mathrm{K}$ was added prior to soil tillage in autumn. Nitrogen was applied in an amount of $67 \mathrm{~kg} \mathrm{ha}^{-1}$ _ Gat and $63 \mathrm{~kg} \mathrm{ha}{ }^{-1}$-Cacinci in the form of urea $(46 \% \mathrm{~N})$. The rest of the $\mathrm{N}$ was applied in two split topdressing applications in the form of CAN (27\% N, calcium ammonium nitrate).

The first topdressing was performed at the beginning of March, at the beginning of the tillering stage of winter wheat (on 5 March at Gat site and on 8 March at Cacinci site) because of the excess precipitation in November (especially at the Gat location) which caused slower and uneven wheat emergence. The second topdressing was performed at the beginning of April at both locations (on 4 April at Gat site and on 6 April at Cacinci site), at the beginning of the stem elongation of winter wheat.

The winter wheat "Srpanjka" (cultivar of Agricultural Institute Osijek) was sown with targeted seed germination number $\left(650 \mathrm{~m}^{-2}\right)$ at the depth 3-4 cm with an inter-row space of $12.5 \mathrm{~cm}$ with wheat seeder right after pre-sowing preparation at both experimental sites (21 October-Gat and 26 October-Cacinci).

Before machine harvest, plant samples were collected using 4 randomly placed frames $(50 \mathrm{~cm} \times 50 \mathrm{~cm})$ on each treatment in three repetitions. Grain separation was performed manually and grain yield was recalculated by the area of ha with grain yield moisture at $12 \%$. Winter wheat harvest was carried out on July 10 (Gat) and July 12 (Cacinci) by combine harvester.

Chemical weed control was carried out in the winter wheat tillering stage (BBCH 26) by applying selective combine postemergence herbicide (amidosulphuron-100 $\mathrm{g} \mathrm{l}^{-1}$ and jodsulphuron methyl-25 $\mathrm{g}^{-1}$ ) in a dose of $0.151 \mathrm{ha}^{-1}$ at both locations.

\subsection{Soil Analysis}

Soil samples for soil fertility control were collected from experimental sites at a depth of 0-30 cm and prepared for laboratory analyses. The standard ISO 11,464 procedure was used to prepare soil samples for physiochemical analyses [33]. Soil pH was measured in a 1:5 (w/v) suspension of soil in $\mathrm{H}_{2} \mathrm{O}$ and in $1 \mathrm{~mol} \mathrm{dm}^{-3} \mathrm{KCl}$ suspension, respectively, using a pH meter (WTW InoLab-IDS Multi 9420) according to the ISO 10,390 standard method [34]. The extraction of AL P and AL K was performed using ammonium lactate-acetic acid extractant, as described by Egner et al. [35], followed by spectrophotometric and flame photometric analyses, respectively. The method prescribed by ISO 14,235 was used to estimate soil organic matter content through the determination of organic carbon (C) by sulphocromic oxidation [36]. The potential acidity of the soil (hydrolytic acidity) was determined by titration, where an alkaline hydrolytic salt (Ca-acetate) to exchange $\mathrm{H}^{+}$and $\mathrm{Al}^{3+}$ ions from the soil adsorption complex was used [37].

\subsection{Weed Population Assesment}

Weediness was determined with a standard procedure by counting and aboveground biomass measurement. Weed density was established twice on each experimental site by using a frame $(0.5 \times 0.5 \mathrm{~m})$ at four randomly selected positions within each plot. All weed species inside the frame were counted and determined, for the first time at the end of March (BBCH 29-end of tillering), and for the second time in July (BBCH 87-ripening), before winter wheat harvesting. Total weed aboveground biomass was evaluated by cutting all weed species at soil level in each frame.

Plant samples were collected in a plastic bags and weighted to determine fresh biomass, and then dried at $60{ }^{\circ} \mathrm{C}$ for $48 \mathrm{~h}$ and weighted for moisture content estimation.

\subsection{Data Analyses}

All collected data were statistically processed by the package Statistica TIBCO Software Inc. Palo Alto, CA, USA [38] in order to establish statistical analyses for all investigated parameters. The influence of different biochar treatments and different levels of NPK fertilization on weed density, weed fresh weight, weed dry weight and winter wheat yield 
at different locations was tested by factorial ANOVA, and the factors were location, biochar treatment and fertilization level. The means were compared by F-test protected LSD values, calculated for $p<0.05$.

\section{Results}

\subsection{Soil Properties}

The determined soil types at the investigated sites were Gleysol (Gat) and Stagnosol (Cacinci) according to the WRB soil classification [30]. The initial soil analysis prior to the experiment determined a strongly acidic reaction of the soil at the Gat site and a very strongly acidic reaction at the site Cacinci [32] (Table 1). The measured values of soil organic matter content (OM) were below $2.5 \%$ at both sites, which indicates a low content of organic matter, while the highest hydrolytic acidity was measured at the Cacinci site

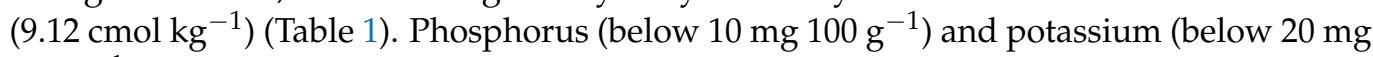
$\left.100 \mathrm{~g}^{-1}\right)$ content were low at both locations [32].

\subsection{Weed Species Composition}

In winter wheat, a total of 17 weed species were determined for both investigated localities (Table 4), and almost 50\% were annual broadleaf weed species. The number of species was almost equal at both localities (12-Gat and 13-Cacinci). The most dominant weed species were Ambrosia artemisiifolia L. and Polygonum aviculare L., followed by Setaria glauca (L.) PB., Echinochloa crus-galli (L.) PB. and Chenopodium album (L.). Specific weed species at the Gat site were Stellaria media L., Setaria viridis L. PB., Plantago major L. and Rumex acetosella L., while specific weed species identified at the Cacinci site were Matricaria chamomilla L., Oxalis fontana Bunge, Apera spica-venti (L.) PB., Rubus caesius L. and Agropyron repens (L.) PB. (Table 4). The most numerous weed species at the Cacinci site was Ambrosia artemisiifolia while at Gat the most common was Polygonum aviculare (data not shown).

Table 4. Weed species composition at experimental sites Gat and Cacinci.

\begin{tabular}{|c|c|c|c|c|}
\hline Annual Broadleaf & Common Name & EPPO Code & Gat & Cacinci \\
\hline Ambrosia artemisiifolia $\mathrm{L}$. & Common ragweed & AMBEL & + & + \\
\hline Anagallis arvensis L. & Scarlet pimpernel & ANGAR & + & + \\
\hline Chenopodium album $\mathrm{L}$. & $\begin{array}{c}\text { Common } \\
\text { lambsquarters }\end{array}$ & CHEAL & + & + \\
\hline Matricaria chamomilla L. & Chamomile & МATCH & - & + \\
\hline Oxalis fontana Bunge & Lemon clover & OXAST & - & + \\
\hline Polygonum aviculare L. & Common knotweed & POLPE & + & + \\
\hline Stellaria media (L.) Vill. & Chickweed & STEME & + & - \\
\hline Veronica hederifolia L. & Ivyleaf speedwell & VERHE & + & + \\
\hline \multicolumn{5}{|l|}{ Annual grasses } \\
\hline Apera spica-venti (L.) PB. & Common windgrass & APESV & - & + \\
\hline Echinochla crus-galli (L.) PB. & Barny grass & ECHCG & + & + \\
\hline Setaria glauca (L.) PB. & Pearl millet & SETPU & + & + \\
\hline Setaria viridis L. PB. & Green foxtail & SETVI & + & - \\
\hline \multicolumn{5}{|l|}{ Perennial broadleaf } \\
\hline Convolvulus arvensis L. & Bidweed & CONAR & + & + \\
\hline Plantago major L. & Broadleaf plantain & PLAMA & + & - \\
\hline Rumex acetosella L. & Sheep sorrel & RUMAA & + & - \\
\hline Rubus caesius L. & European dewberry & RUBCA & - & + \\
\hline \multicolumn{5}{|l|}{ Perennial grasses } \\
\hline Agropyron repens (L.) PB. & Quackgrass & AGRRE & - & + \\
\hline
\end{tabular}

(+) weed species present, (-) weed species not present 


\subsection{Weed Incidence in Winter Wheat Late Tillering Stage}

All investigated weed parameters were higher at the Gat experimental site. Statistically significant differences $(p<0.05)$ were found in terms of weed density and weed fresh and dry weight. Weed density and weed fresh weight were about $60 \%$ higher at the Gat site when compared with the Cacinci site (Table 5), while the measured dry weight of weeds was $40 \%$ higher (Table 5).

Table 5. Biochar and fertilization effects on weed density, weed fresh and weed dry weight at two locations at the end of the winter wheat tillering stage.

\begin{tabular}{|c|c|c|c|c|c|c|c|c|c|c|c|c|}
\hline \multirow[t]{2}{*}{ TB } & \multicolumn{2}{|c|}{$\begin{array}{l}\text { Weed Density } \\
\qquad\left(\mathrm{m}^{-2}\right)\end{array}$} & \multirow[t]{2}{*}{$A v B$} & \multirow[t]{2}{*}{ SD } & \multicolumn{2}{|c|}{$\begin{array}{c}\text { Weed Fresh } \\
\text { Weight }\left(\mathrm{g} \mathrm{m}^{-2}\right)\end{array}$} & \multirow[t]{2}{*}{ AvB } & \multirow[t]{2}{*}{ SD } & \multicolumn{2}{|c|}{$\begin{array}{c}\text { Weed Dry } \\
\text { Weight }\left(\mathrm{g} \mathrm{m}^{-2}\right)\end{array}$} & \multirow[t]{2}{*}{ AvB } & \multirow[t]{2}{*}{ SD } \\
\hline & F0 & F1 & & & F0 & F1 & & & F0 & F1 & & \\
\hline \multicolumn{13}{|c|}{ Gat (L) } \\
\hline B0 & 98.66 & 44.00 & $71.33^{\mathrm{A}}$ & 30.66 & 101.97 & 20.04 & $61.01^{\mathrm{B}}$ & 2.75 & 42.61 & 12.31 & $27.46^{\mathrm{B}}$ & 16.75 \\
\hline B1 & 91.33 & 60.66 & $76.00^{\mathrm{A}}$ & 17.15 & 163.50 & 34.88 & $99.19^{\mathrm{A}}$ & 11.70 & 53.57 & 13.56 & $33.57^{\mathrm{A}}$ & 21.97 \\
\hline B2 & 94.00 & 6.00 & $50.00^{\mathrm{A}}$ & 48.23 & 65.28 & 26.80 & $46.04^{C}$ & 3.64 & 34.51 & 13.66 & $24.08^{C}$ & 11.60 \\
\hline B3 & 20.00 & 4.33 & $12.16^{C}$ & 9.21 & 13.34 & 14.13 & $13.74^{\mathrm{D}}$ & 4.54 & 11.25 & 10.24 & $10.74^{\mathrm{D}}$ & 2.18 \\
\hline $\mathrm{AvF}$ & $76.00^{\mathrm{A}}$ & $28.75^{\text {B }}$ & & & $86.02^{\mathrm{A}}$ & $23.96^{\mathrm{B}}$ & & & $35.48^{\mathrm{A}}$ & $12.44^{\mathrm{B}}$ & & \\
\hline SD & 34.00 & 25.85 & & & 57.32 & 8.58 & & & 16.37 & 2.26 & & \\
\hline $\mathrm{AV}(\mathrm{L})$ & \multicolumn{2}{|c|}{$52.37^{\mathrm{A}}$} & & & \multicolumn{2}{|c|}{$54.99^{\mathrm{A}}$} & & & \multicolumn{2}{|c|}{$23.96^{\mathrm{A}}$} & & \\
\hline SD & \multicolumn{2}{|c|}{38.14} & & & \multicolumn{2}{|c|}{51.10} & & & \multicolumn{2}{|c|}{16.40} & & \\
\hline \multicolumn{13}{|c|}{ Cacinci (L) } \\
\hline B0 & 15.33 & 17.33 & $16.33^{\mathrm{A}}$ & 3.66 & 17.14 & 18.76 & $17.95^{\mathrm{B}}$ & 45.00 & 12.62 & 12.98 & $12.80^{\mathrm{A}}$ & 1.27 \\
\hline B1 & 21.66 & 18.33 & $20.00^{\mathrm{A}}$ & 2.09 & 20.35 & 39.92 & $30.13^{A}$ & 70.72 & 13.81 & 18.00 & $15.90^{\mathrm{A}}$ & 4.17 \\
\hline B2 & 12.33 & 28.66 & $20.50^{\mathrm{A}}$ & 9.75 & 18.13 & 19.24 & $18.69^{\mathrm{B}}$ & 21.23 & 14.23 & 13.82 & $14.00^{\mathrm{A}}$ & 3.75 \\
\hline B3 & 15.33 & 24.00 & $19.66^{\mathrm{A}}$ & 5.71 & 19.43 & 25.56 & $22.49^{\mathrm{B}}$ & 2.70 & 13.72 & 15.10 & $14.41^{\mathrm{A}}$ & 2.07 \\
\hline AvF & $16.16^{\mathrm{B}}$ & $22.08^{\mathrm{A}}$ & & & $18.76^{\mathrm{B}}$ & $25.87^{\mathrm{A}}$ & & & $13.59 \mathrm{~A}$ & $14.97 \mathrm{~A}$ & & \\
\hline SD & 4.19 & 5.94 & & & 2.88 & 9.80 & & & 2.83 & 3.26 & & \\
\hline $\mathrm{AV}(\mathrm{L})$ & \multicolumn{3}{|c|}{$19.12^{B}$} & & \multicolumn{2}{|c|}{$22.31^{\text {B }}$} & & & \multicolumn{2}{|c|}{$14.28^{B}$} & & \\
\hline SD & \multicolumn{2}{|c|}{5.87} & & & \multicolumn{2}{|c|}{7.94} & & & \multicolumn{2}{|c|}{3.07} & & \\
\hline $\mathrm{AV}(\mathrm{F})$ & $46.08^{\mathrm{A}}$ & $25.41^{\text {B }}$ & & & $52.39^{\mathrm{A}}$ & $24.92^{B}$ & & & $25.54^{\mathrm{A}}$ & $13.71^{B}$ & & \\
\hline \multirow[t]{5}{*}{ SD } & 38.67 & 18.66 & & & 52.49 & 9.06 & & & 16.03 & 3.03 & & \\
\hline & \multirow{4}{*}{\multicolumn{3}{|c|}{$\begin{array}{c}\mathbf{F}_{\mathrm{L}}=\mathbf{7 7 8 . 4 8 9 , p}=0.000000 \\
\mathrm{~F}_{\mathrm{F}}=\mathbf{3 0 0 . 7 5 3 , p}=0.000000 \\
\mathrm{~F}_{\mathrm{LXB}}=156.396, p=0.000000 \\
\mathrm{~F}_{\mathrm{LXF}}=497.609, p=0.000000\end{array}$}} & & \multicolumn{4}{|c|}{$\mathbf{F}_{\mathbf{L}}=\mathbf{7 0 2 . 0 2 4 , p = 0 . 0 0 0 0 0 0}$} & \multicolumn{4}{|c|}{$\mathbf{F}_{\mathbf{L}}=\mathbf{1 4 8 . 9 4 3 , p = 0 . 0 0 0 0 0 0}$} \\
\hline & & & & & \multicolumn{4}{|c|}{$\mathrm{F}_{\mathrm{F}}=496.318, p=0.000000$} & \multicolumn{4}{|c|}{$\mathbf{F}_{\mathbf{F}}=\mathbf{1 8 6 . 5 8 5}, p=0.000000$} \\
\hline & & & & & $\mathrm{F}_{\mathrm{L} \lambda}$ & $=174.00$ & $p=0.00$ & & & $3=35.34$ & $p=0.00$ & \\
\hline & & & & & $\mathrm{F}_{\mathrm{L} \gamma}$ & $=786.18$ & $p=0.00$ & & & $=237.0$ & $3, p=0.0$ & \\
\hline
\end{tabular}

TB: Biochar treatments (B0-0 tha ${ }^{-1}$, B1-5 tha ${ }^{-1}$, B2-10 tha ${ }^{-1}$, B3-15 tha ${ }^{-1}$ ), F0—without fertilization, F1—optimal fertilization, $\mathrm{F}_{\mathrm{L}}$-F-test for location, $\mathrm{F}_{\mathrm{F}}-\mathrm{F}$-test for fertilization, $\mathrm{F}_{\mathrm{LXB}}-$ F-test for biochar treatments and location interactions, $\mathrm{F}_{\mathrm{LXF}}-\mathrm{F}$-test for fertilization treatment and location interactions, $p-p$ value, AvB - average value of biochar at specific location, AvF-average value of fertilization at specific location, $\mathrm{AV}(\mathrm{L})$ - average value of location, $\mathrm{AV}(\mathrm{F})$ - average value of fertilization, SD—standard deviation. Values within the same column/row followed by different capital letters are statistically different using the protected least significant difference (LSD) test at $p<0.05$.

Fertilization, on average, significantly affected the occurrence of weeds $(p<0.05)$ Weediness was lower in fertilization treatments compared to non-fertilized (Table 5). Weed density and weed fresh and dry weight were significantly lower $(p<0.05)$ when optimal fertilization was applied (Table 5). Weed fresh weight was more than twice as high in treatments without fertilization, while dry weight was higher for almost $50 \%$. Statistically significant differences among fertilization treatments (F0 and F1) were found on the experimental Gat site, and fertilization significantly affected weed reduction (Table 5). Weed density and weed fresh and weed dry weight were significantly lower $(p<0.05)$ on F1 treatment. Weed density and weed dry weight were almost three times lower when optimal fertilization was applied, when compared to the unfertilized treatment (Table 5). 
Unlike the mentioned location, at the Cacinci site, fertilization caused an increase in weediness in terms of weed density and fresh weight of weeds, while in the case of weed dry weight, statistically significant differences were not recorded (Table 5). At the Gat site, an increase in the dose of biochar led to a decrease in weed density, with significant differences compared to the control (B0) as well as weed fresh and dry weight on site (Table 5). At the Cacinci site, treatment B1 significantly increased weed fresh weight compared to the control (B0).

According to the obtained results, different biochar treatments, on average, affected weed parameters in the winter wheat tillering stage. Statistically significant differences $(p<0.05)$ were found for all weed parameters and among all applied doses of biochar, with the exception of the dry weight of weeds, where there were no significant differences among treatments B2 and B0 (control) (Figure 3).

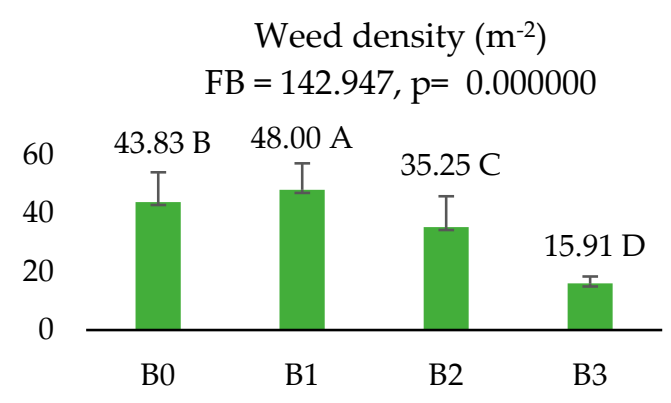

(a)

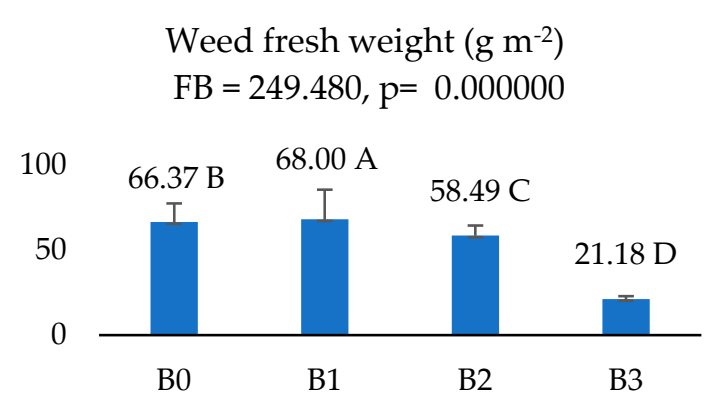

(b)

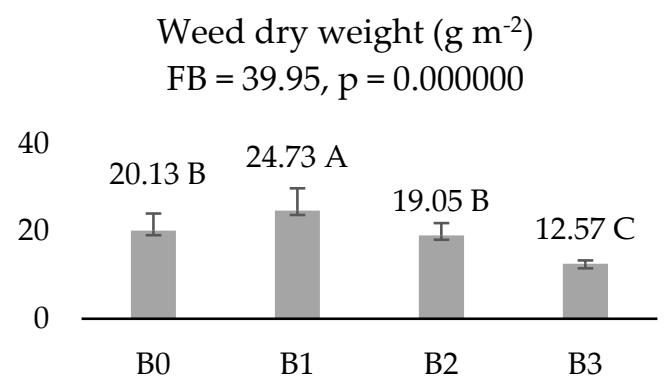

(c)

Figure 3. Influence of biochar treatments (B0-0 $t \mathrm{ha}^{-1}, \mathrm{~B} 1-5 \mathrm{t} \mathrm{ha} \mathrm{a}^{-1}, \mathrm{~B} 2-10 \mathrm{t} \mathrm{ha} \mathrm{a}^{-1}, \mathrm{~B} 3-15 \mathrm{tha} \mathrm{h}^{-1}$ ) on weed density (a), weed fresh weight (b) and weed dry weight (c). FB-F-test for biochar treatments, $p-p$ value. Columns marked with different capital letters are statistically different using the protected least significant difference (LSD) test at $p<0.05$.

For treatment B1, weed density and weed fresh and dry weight were the highest compared to other biochar treatments and the control. Increasing the dose of biochar led to a decrease in weediness. Treatment B3 resulted in the smallest weed density and weed fresh and dry weight (Figure 3).

Significant interactions $(p<0.05)$ were recorded regarding combined applications of different biochar amounts and optimal fertilization (Figure 4).

The lowest weed density was recorded for treatment B3 with optimal fertilization. Biochar treatment B1 resulted in an increased weed density with optimal fertilization, as well as weed fresh weight (Figure 4). Treatment B3 recorded the smallest weed fresh and weed dry weight when applied without optimal fertilization. 
Weed density $\left(\mathrm{m}^{-2}\right)$, weed fresh weight and weed dry weight (g $\mathrm{m}^{-2}$ ) in the wheat late tillering stage

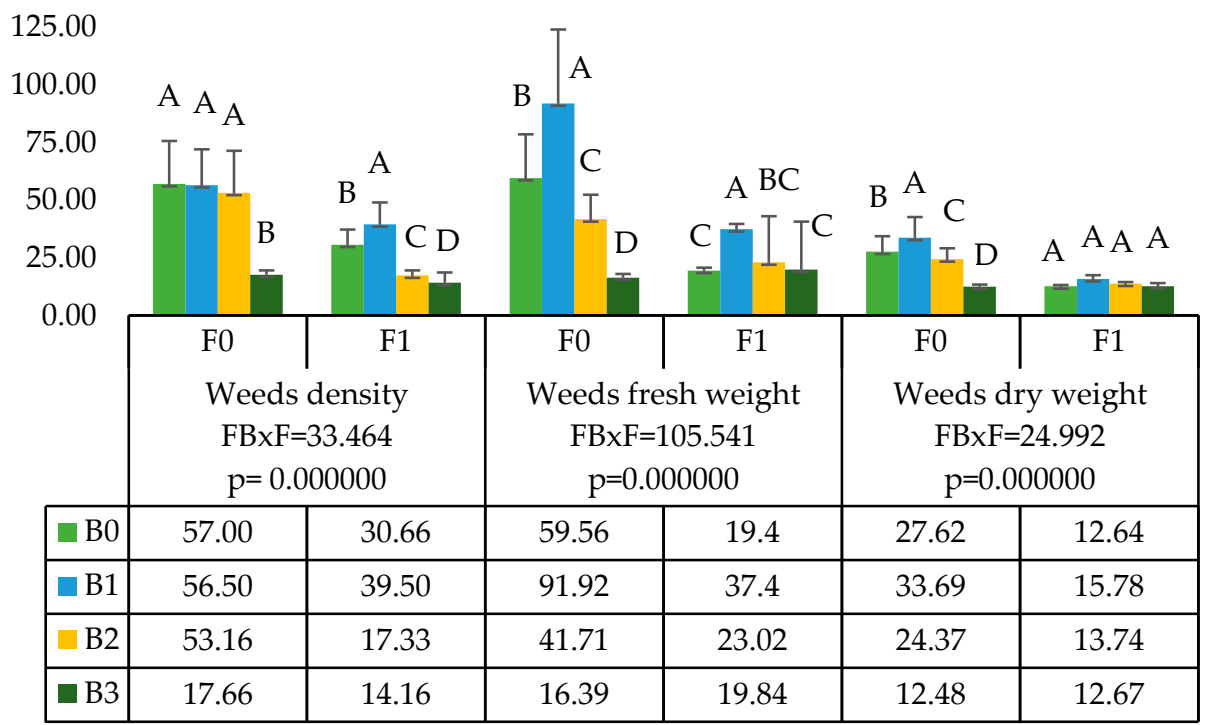

Figure 4. The influence of biochar (B0-0 $\mathrm{t} \mathrm{ha}^{-1}, \mathrm{~B} 1-5 \mathrm{t} \mathrm{ha} \mathrm{h}^{-1}, \mathrm{~B} 2-10 \mathrm{t} \mathrm{ha} \mathrm{h}^{-1}, \mathrm{~B} 3-15 \mathrm{t} \mathrm{ha} \mathrm{h}^{-1}$ ) and fertilization (F0-without fertilization, $\mathrm{F} 1$-optimal fertilization) interactions on weed incidence. FBXF-F-test for biochar-fertilization interaction, $p-p$ value. Columns marked with different capital letters are statistically different using the protected least significant difference (LSD) test at $p<0.05$.

\subsection{Weed Incidence in the Winter Wheat Ripening Stage}

All measured weed indicators were higher at the Cacinci site compared to Gat. Statistically significant differences were determined between sites for weed density, weed fresh weight and weed dry weight (Tables 6 and 7).

Table 6. Biochar and fertilization effects on weed density $\left(\mathrm{m}^{-2}\right)$.

\begin{tabular}{|c|c|c|c|c|c|c|c|c|}
\hline \multirow{3}{*}{ TB } & \multicolumn{2}{|c|}{ Gat (L) } & $\mathbf{A v} \mathbf{v}_{\mathbf{B}}$ & SD & \multicolumn{2}{|c|}{ Cacinci (L) } & $\mathbf{A v} \mathbf{v}_{\mathbf{B}}$ & SD \\
\hline & \multicolumn{8}{|c|}{ Weed Density $\left(\mathrm{m}^{-2}\right)$} \\
\hline & F0 & F1 & & & F0 & F1 & & \\
\hline B0 & 264.66 & 229.33 & $247.00^{\mathrm{A}}$ & 33.43 & 375.33 & 287.33 & $331.33^{C}$ & 51.95 \\
\hline B1 & 256.00 & 124.66 & $190.33^{\mathrm{B}}$ & 75.23 & 352.00 & 335.33 & $343.66^{C}$ & 25.43 \\
\hline B2 & 164.00 & 176.00 & $170.00^{\mathrm{B}}$ & 23.83 & 441.33 & 321.33 & $381.33^{\mathrm{B}}$ & 67.79 \\
\hline B3 & 26.00 & 135.33 & $80.66^{\mathrm{C}}$ & 60.39 & 511.33 & 537.33 & $524.33^{\mathrm{A}}$ & 19.89 \\
\hline$A v_{F}$ & $177.66^{\mathrm{A}}$ & $166.33^{\mathrm{A}}$ & & & $420.00^{\mathrm{A}}$ & $370.33^{\mathrm{B}}$ & & \\
\hline SD & 102.35 & 47.48 & & & 65.65 & 104.92 & & \\
\hline $\operatorname{AV}(\mathrm{L})$ & \multicolumn{2}{|c|}{$172.00^{\mathrm{B}}$} & \multicolumn{6}{|c|}{$395.166^{\mathrm{A}}$} \\
\hline SD & \multicolumn{2}{|c|}{78.24} & \multicolumn{6}{|c|}{89.27} \\
\hline $\mathrm{AV}(\mathrm{F})$ & \multirow{2}{*}{\multicolumn{2}{|c|}{$\begin{array}{c}\mathbf{F 0}=298.83^{\mathrm{A}} \\
149.63\end{array}$}} & \multirow{2}{*}{\multicolumn{6}{|c|}{$\begin{array}{c}\mathbf{F 1}=\mathbf{2 6 8 . 3 3 ^ { \mathbf { B } }} \\
131.14\end{array}$}} \\
\hline SD & & & & & & & & \\
\hline \multicolumn{9}{|c|}{$\begin{array}{c}\mathbf{F}_{\mathbf{L}}=1 \mathbf{1 8 6 . 4 3 2}, p=0.000000 \\
\mathbf{F}_{\mathbf{F}}=\mathbf{2 2 . 1 6 1}, p=0.000046 \\
\mathrm{~F}_{\mathrm{LXB}}=144.744, p=0.000000 \\
\mathrm{~F}_{\mathrm{LXF}}=8.751, p=0.005777\end{array}$} \\
\hline
\end{tabular}

TB: Biochar treatments (B0-0 $\mathrm{t} \mathrm{ha}^{-1}, \mathrm{~B} 1-5 \mathrm{t} \mathrm{ha}^{-1}$, B2-10 $\mathrm{t} \mathrm{ha}^{-1}$, B3-15 tha ${ }^{-1}$ ), F0—without fertilization, F1-optimal fertilization, $\mathrm{F}_{\mathrm{L}}-\mathrm{F}-\mathrm{test}$ for location, $\mathrm{F}_{\mathrm{F}}-\mathrm{F}$-test for fertilization, $\mathrm{F}_{\mathrm{LXB}}-$ F-test for biochar treatment and location interactions, $\mathrm{F}_{\mathrm{LXF}}-\mathrm{F}$-test for fertilization treatment and location interactions. $\mathrm{AV}(\mathrm{L})$ - average value of location, $\mathrm{AV}(\mathrm{F})$-average value of fertilization, $\mathrm{A}_{\mathrm{VB}}$ - average values of biochar inside location, $\mathrm{A}_{\mathrm{VF}}$ - average values of fertilization inside location, $\mathrm{SD}$ - standard deviation, $p-p$ value. Values within the same column/row followed by different capital letters are statistically different using the protected least significant difference (LSD) test at $p<0.05$. 
Table 7. Biochar and fertilization effects on weed fresh and dry weight.

\begin{tabular}{|c|c|c|c|c|c|c|c|c|}
\hline \multirow[t]{2}{*}{ ТВ } & \multicolumn{2}{|c|}{$\begin{array}{c}\text { Weed Fresh } \\
\text { Weight }\left(\mathrm{g} \mathrm{m}^{-2}\right)\end{array}$} & \multirow[t]{2}{*}{$\mathbf{A v}_{\mathbf{B}}$} & \multirow[t]{2}{*}{ SD } & \multicolumn{2}{|c|}{$\begin{array}{c}\text { Weed Dry } \\
\text { Weight }\left(\mathrm{gm}^{-2}\right)\end{array}$} & \multirow[t]{2}{*}{$\mathbf{A}_{\mathrm{VB}}$} & \multirow[t]{2}{*}{ SD } \\
\hline & F0 & F1 & & & F0 & F1 & & \\
\hline \multicolumn{9}{|c|}{ Gat (L) } \\
\hline B0 & 107.38 & 88.52 & $97.95^{\mathrm{A}}$ & 12.73 & 29.05 & 40.80 & $34.92^{\mathrm{A}}$ & 8.09 \\
\hline B1 & 85.12 & 57.25 & $71.18^{\mathrm{B}}$ & 17.44 & 45.47 & 40.70 & $43.08^{\mathrm{A}}$ & 8.09 \\
\hline B2 & 45.47 & 49.30 & $47.38^{\mathrm{C}}$ & 10.11 & 20.46 & 29.82 & $25.14^{\mathrm{B}}$ & 6.75 \\
\hline B3 & 14.24 & 48.24 & $31.24^{\mathrm{D}}$ & 19.37 & 7.20 & 29.68 & $18.48^{\mathrm{B}}$ & 12.56 \\
\hline$A v_{F}$ & $63.05^{\mathrm{A}}$ & $60.83^{\mathrm{A}}$ & & & $25.56^{\mathrm{B}}$ & $35.25^{\mathrm{A}}$ & & \\
\hline SD & 38.36 & 18.38 & & & 14.89 & 8.39 & & \\
\hline $\mathrm{AV}(\mathrm{L})$ & \multicolumn{2}{|c|}{$61.94^{B}$} & & & \multicolumn{2}{|c|}{$30.41^{B}$} & & \\
\hline SD & \multicolumn{2}{|c|}{29.44} & & & \multicolumn{2}{|c|}{12.81} & & \\
\hline \multicolumn{9}{|c|}{ Cacinci (L) } \\
\hline B0 & 158.88 & 141.24 & $149.91^{\mathrm{C}}$ & 17.64 & 79.27 & 66.30 & $72.78^{\mathrm{B}}$ & 11.48 \\
\hline B1 & 159.91 & 121.24 & $140.58^{\mathrm{D}}$ & 28.02 & 66.74 & 61.12 & $63.93^{B}$ & 13.83 \\
\hline B2 & 214.58 & 157.24 & $185.91^{\mathrm{B}}$ & 34.21 & 105.50 & 66.94 & $86.22^{\mathrm{A}}$ & 22.52 \\
\hline B3 & 198.58 & 221.24 & $209.91^{\mathrm{A}}$ & 14.67 & 91.54 & 93.38 & $92.46^{\mathrm{A}}$ & 8.26 \\
\hline$A v_{F}$ & $182.91^{\mathrm{A}}$ & $160.24^{\mathrm{B}}$ & & & $85.76^{A}$ & $71.93^{B}$ & & \\
\hline $\mathrm{SD}$ & 30.01 & 40.51 & & & 17.99 & 15.89 & & \\
\hline $\mathrm{AV}(\mathrm{L})$ & \multicolumn{2}{|c|}{$171.58^{\mathrm{A}}$} & & & \multicolumn{2}{|c|}{$78.85^{\mathrm{A}}$} & & \\
\hline SD & \multicolumn{2}{|c|}{36.74} & & & \multicolumn{2}{|c|}{18.04} & & \\
\hline $\mathrm{AV}(\mathrm{F})$ & $122.98^{A}$ & $110.53^{B}$ & & & 55.66 & 53.59 & & \\
\hline \multirow[t]{2}{*}{ SD } & 69.87 & 59.37 & & & 34.73 & 22.48 & & \\
\hline & \multicolumn{3}{|c|}{$\begin{array}{c}\mathbf{F}_{\mathbf{L}}=872.989, p=0.000000 \\
F_{F}=11.250, p=0.002061 \\
F_{L X B}=63.828, p=0.000000 \\
F_{L X F}=7.587, p=0.009616\end{array}$} & & \multicolumn{3}{|c|}{$\begin{array}{c}\mathbf{F}_{\mathbf{L}}=359.495, p=0.000000 \\
\mathbf{F}_{\mathbf{F}}=\mathbf{0 . 6 5 7 n s}, p=0.423627 \\
F_{\mathrm{LXB}}=4.727, p=0.000000 \\
\mathrm{~F}_{\mathrm{LXF}}=21.177, p=0.000063\end{array}$} & \\
\hline
\end{tabular}

TB: Biochar treatments (B0-0 t ha ${ }^{-1}, \mathrm{~B} 1-5 \mathrm{t} \mathrm{ha}^{-1}, \mathrm{~B} 2-10 \mathrm{t} \mathrm{ha}^{-1}$, B3-15 tha ${ }^{-1}$ ), F0—without fertilization, F1—optimal fertilization, $\mathrm{F}_{\mathrm{L}}$-F-test for location, $\mathrm{F}_{\mathrm{F}}-\mathrm{F}$-test for fertilization, $\mathrm{F}_{\mathrm{LXB}}-\mathrm{F}$-test for biochar treatment and location interactions, $\mathrm{F}_{\mathrm{LXF}}-\mathrm{F}$-test for fertilization treatment and location interactions. $\mathrm{AV}(\mathrm{L})$ - average values of location, $\mathrm{AV}(\mathrm{F})$ - average values of fertilization, $\mathrm{A}_{\mathrm{VB}}$ - average values of biochar inside location, $\mathrm{A}_{\mathrm{VF}}$ - average values of fertilization inside location, $\mathrm{SD}$-standard deviation, $p$ - $p$ value. Values within the same column/row followed by different capital letters are statistically different using the protected least significant difference (LSD) test at $p<0.05$.

Weed density was more than twice as high at the Cacinci site in comparison to the Gat site (Table 6). Weed fresh weight was almost three times higher, while the dry mass was increased by almost $60 \%$ at the Cacinci site (Table 7 ).

Fertilization, on average, significantly affected $(p<0.05)$ weed density and weed fresh weight. Both parameters were statistically significantly lower, with optimal fertilization (Tables 6 and 7), in contrast to the dry weight, where the effect of fertilization was not determined. Fertilization reduced weed incidence at the Cacinci site (Tables 6 and 7). Weed density, weed fresh weight and weed dry weight were significantly lower $(p<0.05)$ when optimal fertilization was applied compared to F0. At the Gat site, fertilization significantly increased the fresh mass of weeds compared to F0 (Table 7), while it had no significant effect on weed density and weed fresh weight (Tables 6 and 7).

The application of biochar affected weeds at individual experimental sites. At the Gat site, all biochar treatments significantly reduced weed density and weed fresh weight compared to B0 (Tables 6 and 7). The lowest weed density and weed fresh weight were recorded for the $\mathrm{B} 3$ treatment, with values about three times smaller compared to the control treatment B0. Weed dry weight was significantly lower for B2 and B3 treatments compare to the control (B0) (Tables 6 and 7). Unlike the Gat site, at the Cacinci site the application of biochar led to an increase in weediness. The highest weed density, weed fresh weight and weed dry weight were recorded for the B3 treatment with significant differences compared to the control (B0) (Tables 6 and 7). At the Cacinci site, the recorded 
weed density and weed dry weight for treatment B1 were not significantly different with regard to the control. The measured weed fresh weight was the lowest for the B1 treatment and was significantly different compared to the control, B0 (Table 7).

Different biochar treatments, on average, significantly affected $(p<0.05)$ weed occurrence in the winter wheat ripening stage. Biochar treatment B1 resulted in the lowest weed density and weed fresh weight (Figure 5), with significant differences compared to the control (B0). Weed dry weight showed no response to different applied amounts of biochar. Higher doses of biochar in the ripening stage of winter wheat led to an increase in weed density and weed fresh weight (Figure 5), where treatment B3 recorded the highest values of these parameters, although without statistically significant differences compared to B0.

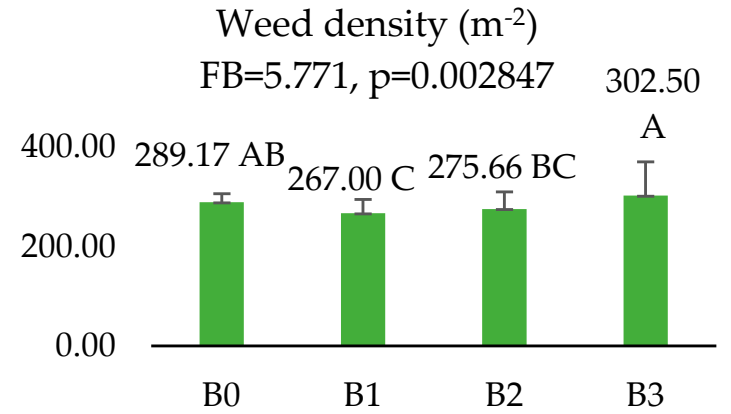

(a)
Weed fresh weight $\left(\mathrm{g} \mathrm{m}^{-2}\right)$

$\mathrm{FB}=4.463, \mathrm{p}=0.009466$

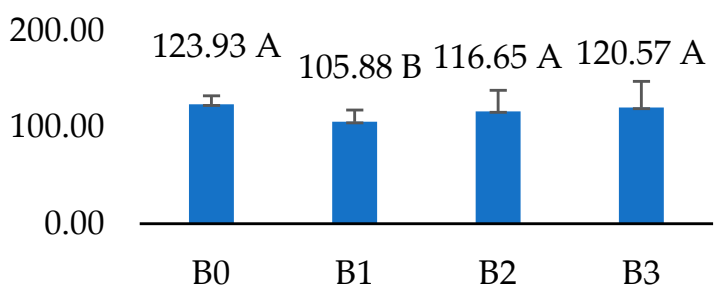

(b)

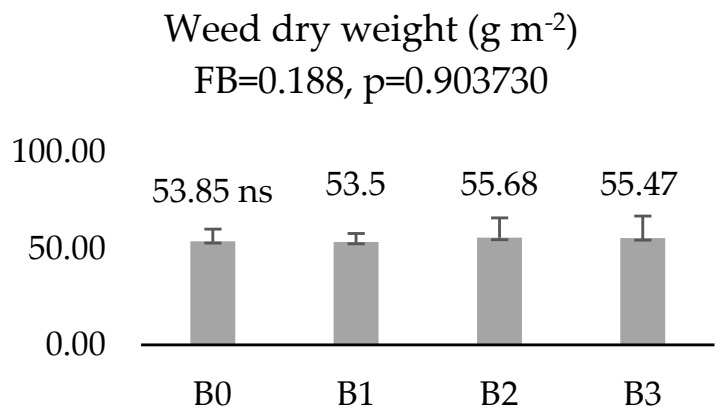

(c)

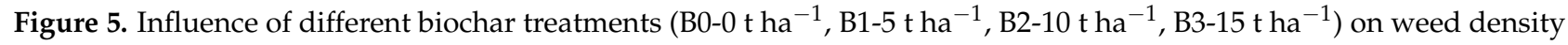
(a), weed fresh weight (b) and weed dry weight (c). FB-F-test for biochar treatments, $p-p$ value. Columns marked with different capital letters are statistically different using the protected least significant difference (LSD) test at $p<0.05$.

Significant interactions $(p<0.05)$ were found among different biochar and fertilization treatments (Figure 6).

The highest dose of biochar, treatment B3, with optimal fertilization caused a significant increase in weed density (Figure 6). The determined weed fresh weight and weed dry weight also increased with the application of the highest dose of biochar. The same biochar treatment resulted in the lowest weeding for all measured parameters when applied without optimal fertilization in the ripening stage of wheat (Figure 6). Treatment B1, applied with optimal fertilization, had the lowest weed density and weed fresh weight, with statistically significant differences confirmed in relation to other biochar treatments applied with fertilization. 
Weed density $\left(\mathrm{m}^{-2}\right)$, weed fresh weight and weed dry weight $(\mathrm{g}$ $\left.\mathrm{m}^{-2}\right)$ in the winter wheat ripening stage

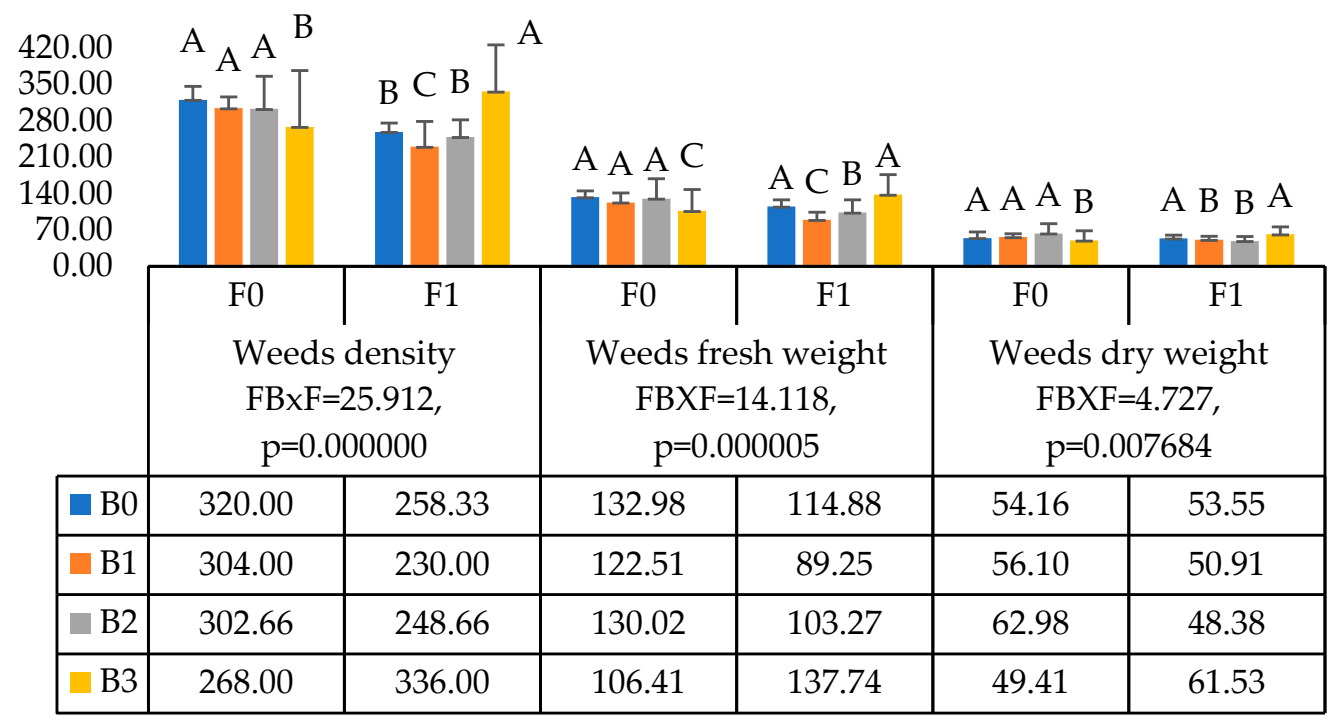

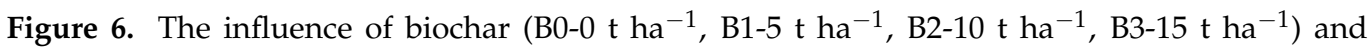
fertilization (F0-without fertilization, F1-optimal fertilization) interactions on weed incidence. FBXF-F-test for biochar-fertilization interaction, $p-p$ value. Columns marked with different capital letters are statistically different using the protected least significant difference (LSD) test at $p<0.05$.

\subsection{Winter Wheat Yield}

The experiment location significantly affected the achieved yields of the winter wheat Table 8). Statistically significant differences $(p<0.05)$ were found between the Gat and Cacinci sites, and a higher yield was achieved at the Gat site (Table 8).

Table 8. Biochar and fertilization effects on winter wheat yield $\left(\mathrm{t} \mathrm{ha}^{-1}\right)$ at the experimental sites Gat and Cacinci.

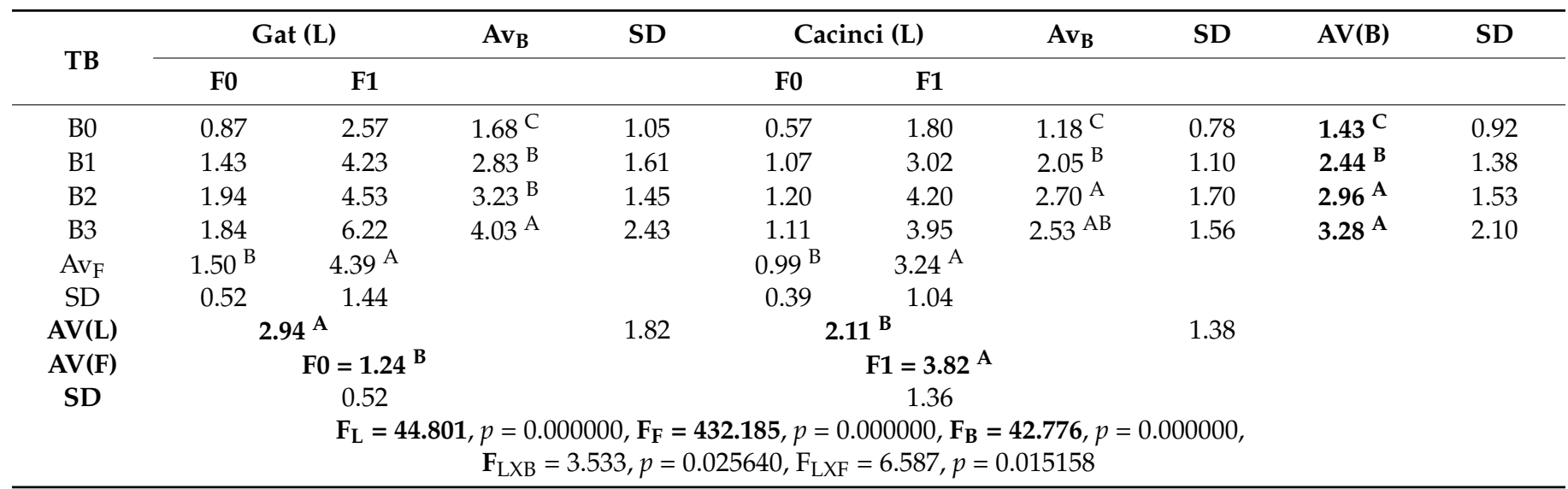

Biochar treatments (B0-0 t ha-1, B1-5 t ha-1, B2-10 t ha-1, B3-15 t ha-1), F0-without fertilization, F1-optimal fertilization, FL-F-test for location, FF-F-test for fertilization, FB-F-test for biochar, $\mathrm{F}_{\mathrm{LXB}}-$ F-test for biochar treatment and location interactions, $\mathrm{F}_{\mathrm{LXF}}-\mathrm{F}$-test for fertilization treatment and location interactions. $\mathrm{AV}(\mathrm{B})$ - average value of bichar, $\mathrm{A}_{\mathrm{VB}}$-average value of biochar inside location, $A_{V F}$-average value of fertilization inside location, $\mathrm{AV}(\mathrm{L})$ - average value of location, $\mathrm{AV}(\mathrm{F})$ —average value of fertilization, $\mathrm{SD}$-standard deviation, $p-p$ value. Values within the same column followed by different capital letters are statistically different using the protected least significant difference (LSD) test at $p<0.05$.

Optimal fertilization led to a statistically significant $(p<0.05)$ increase in winter wheat yield and an on average three times higher yield was achieved with the application of mineral fertilizer. The application of biochar caused a statistically significant $(p<0.05)$ increase in yield compared to the control (B0). The highest yield was achieved with treatment B3, with confirmed statistically significant differences in relation to the control 
and B1 (Table 8). At the Gat site, with treatment B3, an about $60 \%$ higher winter wheat yield was achieved compared to the control. With optimal fertilization at Gat, an almost three times higher winter wheat yield was achieved compared to the non-fertilized treatment (F0), and the increase in yield at Cacinci was almost 70\%. The application of biochar along with optimal fertilization increased winter wheat yield (Figure 7) and a significant interaction $(p<0.05)$ between biochar and fertilization was found. Increasing the dose of biochar with optimal fertilization significantly increased the winter wheat yield (Figure 7). The highest winter wheat yield was achieved by treatment B3 with optimal fertilization.

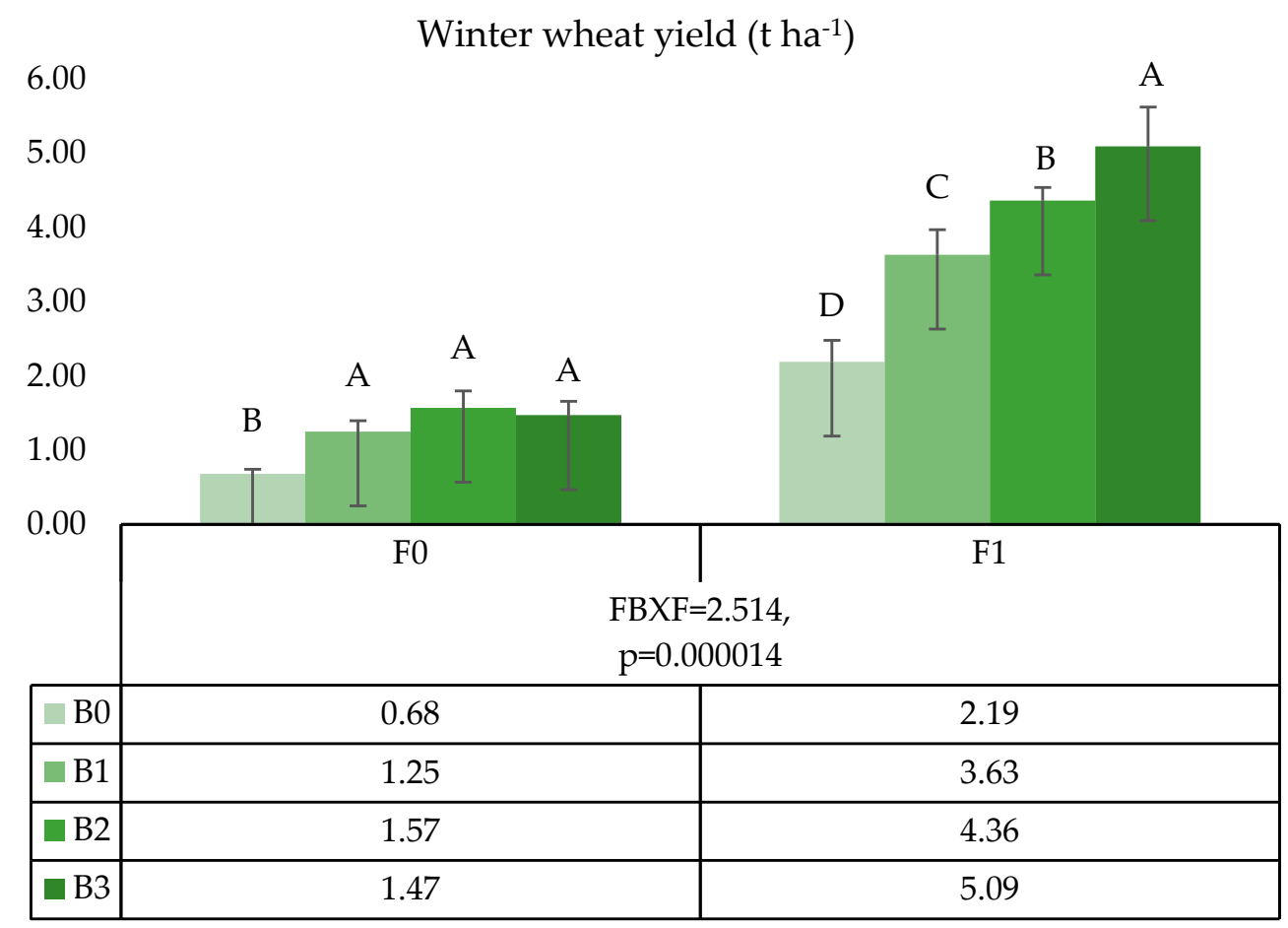

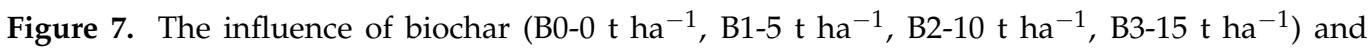
fertilization (F0-without fertilization, F1—optimal fertilization) interactions on winter wheat yield. FBXF-F-test for biochar and fertilization interaction, $p-p$ value. Columns marked with different capital letters are statistically different using the protected least significant difference (LSD) test at $p<0.05$.

\section{Discussion}

\subsection{Basic Soil Properties}

According to the obtained values of basic soil fertility indicators at two experimental sites, Gat (soil type-Gleysol) and Cacinci (soil type-Stagnosol), the presence of certain soil degradation processes can be determined at both localities. This is especially indicated by the low content of organic matter. Values of organic matter below $3 \%$ indicate the existence of degradation processes and a significant possibility of reduced soil fertility [39]. The strongly acidic reaction at the Gat site $\left(\mathrm{pH} \mathrm{H}_{2} 05.12\right)$, with Hy acidity $5.80\left(\mathrm{cmol} \mathrm{kg}^{-1}\right)$, and the very strongly acidic reaction at Cacinci ( $\left.\mathrm{pH} \mathrm{H}_{2} \mathrm{O} 5.00\right)$, with Hy acidity value of $9.12\left(\mathrm{cmol} \mathrm{kg}^{-1}\right)$, indicate the need for liming [32,40]. Additionally, a low content of plant available $\mathrm{P}$ and $\mathrm{K}$ at both locations suggests the need for soil fertility status improvement [32]. It is assumed that the use of biochar as a soil conditioner improved soil fertility indicators at the investigated locations and indirectly affected the winter wheat yield and changes in weed infestation. The application of biochar leads to an increase in the soil reaction and has a liming effect $[5,41]$. As an organic conditioner, it increases the content of organic matter in the soil and ultimately leads to an increase in soil fertility [42]. It is assumed that different soil types, such as Gleysol and Stagnosol, with their different properties, had a different response to the application of biochar. 


\subsection{Weed Species Composition}

At the investigated locations, a typical common weed flora for the wheat community of Eastern Croatia was determined [43,44]. According to the life cycle and the leaf shape, most weed species belonged to annual broadleaf species. The defined weed species in this study can be found on soils of moderate $\mathrm{pH}$ value, while some weeds are indicators of the specific conditions of individual sites. According to the associated bio indicator values (ecological indices) for reactions (soil $\mathrm{pH}$ ), almost all identified weeds can be classified as part of the group which represents weeds that prefer weakly acidic or neutral soils [45]. The presence of these weeds on strongly acidic and very acidic soils in this research can be explained by the great ability of weed species to adapt to various adverse conditions [46]. However, at the Gat site, Rumex acetosella was identified. It is an indicator of a strongly acidic soil, growing predominantly on low-fertility soils with moderate humus content [45]. Apera spica-venti and Rubus caesisus were identified only at the Cacinci site. Apera spica-venti is an indicator of an acidic habitat and can be found predominantly on soils with $\mathrm{pH}$ range 3.5-5.5. Soil properties at the Cacinci site also favor the appearance of Rubus caesius [45], affected by the occasional stronger wetting and drying of the soil. The alternation of dry and wet phases is a characteristic of the Stagnosol soil type [47]. It is assumed that certain weed species appeared due to the specific characteristics of the experimental sites' soil properties.

\subsection{Weed Infestation of Winter Wheat}

The higher weed occurrence in terms of all investigated weed parameters recorded at the Gat site in the late tillering stage of winter wheat is a consequence of specific weather conditions at this locality. The large amount of precipitation recorded in February favored the growth and development of weeds. Additionally, excess precipitation was also present in March, which further favored the incidence of weeds, which were more competitive in relation to winter wheat in the stress conditions caused by excess water in the soil. Any factor that negatively affects the crop and causes the intensification of environmental stress makes weeds more competitive in relation to the crop [48]. Previous research also confirms significant differences in weediness within different field conditions $[49,50]$. The second observation of weeds in the winter wheat ripening stage gave opposite results in terms of weediness at specific locations. Greater weediness was recorded at the Cacinci site. The higher occurrence of weeds at the Cacinci site is probably due to the different composition of the weed flora. Namely, the dominant weed species in Cacinci was Ambrosia artemisiifolia, which is a summer annual weed and a strong competitor. Ambrosia starts to germinate in early spring and continues throughout the summer, which allows it an intense appearance and dominance [51]. In July, when the second sampling of weeds was performed, the spring weeds had already finished their vegetation, and the most numerous weed species was Ambrosia. This weed was also found at the Gat site but with very low intensity. In this study, on average, fertilization caused a reduction in weed infestation. Statistically significant differences were found in terms of weed density and weed fresh weight in both stages of winter wheat, tillering and ripening. In the tillering stage, fertilization also significantly affected weed dry weight. The positive effect of fertilization on weed reduction has been proven by previous research $[23,24,52,53]$. The impact of fertilization on weed reduction is a result of the positive effect of added nutrients on winter wheat development, and of greater competition with weeds. Optimal fertilization leads to optimal crop stand and competition for light with weeds, which ultimately leads to a reduction in weediness [21]. Regarding optimal crop stand, better competition against weeds has been achieved recently by the use of remote sensing and precision farming, but mostly in areas under the management of large agribusiness companies (up to 40,000 ha in total [54]. At the Cacinci site, weed density and weed fresh weight were significantly higher with applied fertilization in the tillering stage. This is probably a consequence of the lack of precipitation in March, which negatively reflected on the growth and development of winter wheat and brought weeds to an advantage. It is known from previous research that 
optimal fertilization can benefit weeds more than crops, because weeds absorb nutrients more successfully than crops and the source and availability of nutrients have a strong influence on weeds [20-22].

Biochar, on average, affected the level of weediness depending on the applied dose in the tillering stage of winter wheat. When applied in the amount of $5 \mathrm{tha}^{-1}$ (B1), it caused an increase in weed density, weed fresh weight and weed dry weight, while higher doses caused a decrease in weediness. By increasing the dose of biochar, weediness decreased, which is in line with the research of Arif et al. [55], in whose study weed in maize was reduced by applying the highest dose of biochar $\left(50 \mathrm{t} \mathrm{ha} \mathrm{a}^{-1}\right)$. Another study highlights the increase in weed density and weed fresh biomass when a lower level of biochar is applied [28], which is also confirmed by our research. The decrease in weediness when adding higher doses of biochar is probably a consequence of the better growth and development of winter wheat due to improved soil conditions. This is probably the reason why at the Gat site, biochar influenced weed reduction in the ripening stage of winter wheat, while in Cacinci, a positive effect was not recorded. The only average positive effects on weeds in the ripening stage of winter wheat resulted from a lower dose of biochar (B1). This is probably a consequence of the different agroecological conditions between experimental sites. The positive effects of biochar on soil properties are highly connected with soil type and climatic factors [56]. The positive impact of biochar on crop development is connected to better nutrient retention and cycling, better water holding, more intense microbiological activity and an increase in organic matter [10,44]. For these reasons, biochar is also recommended for organic farming and it has approval in the organic legislature of the EU (EC regulation 889/2008 and EC implementing regulation 2019/2164), especially where there is no sufficient animal manure production [57-59]. Currently, there are not enough data to recommend a specific dose of biochar that will increase yield and reduce weediness, and some research indicates the positive effects on crop development with amounts of 5-50 tha ${ }^{-1}$ with optimal fertilization [60]. The assumption is that biochar at our investigated sites caused an increase in soil fertility, an increase in soil reaction and an improvement in physical properties and organic matter content which led to better winter wheat development, which made it more competitive with weeds in the tillering stage. This research found a significant interaction between biochar and optimal fertilization that is in contrast with the findings of Ali et al. [27], in whose study the interaction between biochar and fertilization was not confirmed in terms of the impact on changes in weed level. In our research, the highest dose of biochar along with optimal fertilization led to a reduction in weediness, probably due to the better growth and development of winter wheat in the tillering stage. However, in second weed sampling (ripening stage), biochar with fertilization caused an increase in weed density, which is consistent with Major et al. [17], in whose study biochar with inorganic fertilizer caused an increase in weed cover and the number of species. The increase in weediness by adding higher doses of biochar along with fertilization is probably due to the higher amount of nutrients available to weeds in the ripening stage of winter wheat, because at that stage the winter wheat nutrient uptake is mostly completed. Winter wheat absorbs the highest amount of nutrients from the beginning of tillering to earing. After fertilization, the ripening of winter wheat takes place mainly at the cost of nutrient reserves with their transfer (allocation) from the photosynthetically inactive parts of the plant (old leaf) to the ear. For this reason, the competition for nutrients between weeds and winter wheat is expressed in the tillering stage, and in ripening the remaining nutrients are more available to the weeds. Biochar has the potential to improve fertilizer use efficiency through increasing cation exchange capacity (CEC), which directly affects the increase in the soil fertility because of better nutrient retention and improved nutrient adsorption of plant nutrients [6,61]. According to some research, the use of biochar along with fertilizer is recommended to maximize its positive effect on the soil $[25,26]$. 


\subsection{Winter Wheat Yield}

The below-average winter wheat yields achieved in this study are a consequence of unfavorable weather conditions at the studied sites. Because of higher rainfall in September, the sowing date was postponed at the Gat experimental site, while in Cacinci, sowing was delayed due to unfavorable tillage conditions. Significantly lower temperatures in January further slowed the growth and development of winter wheat. The decrease in winter wheat yield was strongly affected by the lack of precipitation (April) in the stem elongation phase at both locations. The drought conditions in Cacinci lasted from March to June, which caused the lower yields at this experimental site, together with a higher level of weediness after the tillering stage of winter wheat. Optimal fertilization resulted in an increase in winter wheat yield, which is to be expected because of the improvement in the nutrient status of the low-fertility soil. The results confirm the limiting factors of unfertilized soil: poor nutrient supply due to low $\mathrm{pH}$ and CEC, and low organic matter content. The significant impact of biochar on the increase in winter wheat yield stems from its positive impact on a number of soil properties [7]. It can be assumed that the physical, chemical and biological properties of the soil were improved at the investigated localities. The positive influence of biochar on the above soil properties was proven by previous research $[4,6,8,9]$. The possible increase in soil reaction, organic matter content and plant available nutrients by adding biochar probably resulted in the increase in the yield of winter wheat in this study. Winter wheat yields were significantly higher with the addition of biochar compared to the control. Previous studies indicate the greatest impact of biochar on yield increase when applied with mineral fertilizers, which was also found in our research [41,62]. The application of biochar with optimal fertilization caused a significant increase in winter wheat yield. Higher winter wheat yields were related to higher applied doses of biochar combined with the optimal amount of mineral fertilizer, which was also confirmed by previous research [63]. Better fertilizer use efficiency with biochar application is caused by improved nutrient retention, reduced nutrient losses, better water holding capacity and the liming effects of biochar $[25,62,63]$.

\section{Conclusions}

The different level of weediness in this research was influenced by the specific agroecological conditions of the studied sites. A positive effect of fertilization on weediness on acidic, low-fertility soils was found. The application of biochar affected the level of weediness differently, depending on the applied amount and specific field conditions. Biochar in an amount of $15 \mathrm{t} \mathrm{ha}^{-1}$ (single application or with optimal fertilization) reduced the weed pressure in the winter wheat tillering stage. Optimal fertilization resulted in the increase in winter wheat yield on low-fertility soils. Winter wheat yield was increased by applying a higher dose of biochar. Biochar applied at a dose of $15 \mathrm{tha}^{-1}$ resulted in the highest winter wheat yield. Combined biochar and fertilizer addition significantly increased winter wheat yield. The response of weeds to the biochar application, alone or with optimal fertilization, differed within the vegetation period and individual fields, but without a final negative impact on yield.

The application of biochar increased the winter wheat yield, which indicates the need for its further use due to its positive effects on numerous soil properties and its importance in climate change mitigation. Further research is needed in different agroecological conditions and crop production systems, which would contribute to existing knowledge about the role of biochar in agricultural production.

Author Contributions: Conceptualization, B.B., I.J., M.R. and D.J.; methodology, B.B., B.Đ., I.J. and M.R.; software, B.S. and I.J.; validation, B.B., I.J., D.J. and B.Đ.; formal analysis, B.B., I.J., D.J., B.S. and B.Đ.; investigation, B.Đ., B.B., D.J. and I.J.; resources, B.Đ. and D.J.; data curation, B.Đ., B.B., D.J. and I.J.; writing-original draft preparation, B.B., B.Đ. and I.J.; writing-review and editing, B.B., I.J., D.J., M.R. and B.S.; visualization, I.J. and B.B.; supervision, I.J. and D.J.; project administration, B.Đ.; funding acquisition, B.Đ. and D.J. All authors have read and agreed to the published version of the manuscript. 
Funding: This research was funded by the Croatian Ministry of Agriculture (VIP Project: "Application of biochar as a soil conditioner - a step towards sustainable crop production", research grant number 2015-13/32).

Institutional Review Board Statement: Not applicable.

Informed Consent Statement: Not applicable.

Data Availability Statement: Most of the collected data are contained in the tables and figures in the manuscript.

Conflicts of Interest: The authors declare no conflict of interest.

\section{References}

1. Liu, C.; Allan, R.P. Observed and simulated precipitation responses in wet and dry regions 1850-2100. Environ. Res. Lett. 2013, 8 , 1-11. [CrossRef]

2. Jug, D.; Jug, I.; Brozović, B.; Vukadinović, V.; Stipešević, B.; Đurđević, B. The role of conservation agriculture in climate change mitigation. Poljoprivreda 2018, 24, 35-44. [CrossRef]

3. Igrejas, G.; Branlard, G. The Importance of Wheat. In Wheat Quality for Improving Processing and Human Health, 1st ed.; Ikeda, T., Igrejas, G., Guzmán, C., Eds.; Springer: Cham, Switzerland, 2020; pp. 1-7. [CrossRef]

4. Lehmann, J.; Rillig, M.C.; Thies, J.; Masiello, C.A.; Hockaday, W.C.; Crowley, D. Biochar effects on soil biota-A review. Soil Biol. Biochem 2011, 43, 1812-1836. [CrossRef]

5. Jeffery, S.; Verheijen, F.G.A.; Van Der Velde, M.; Bastos, A.C. A quantitative review of the effects of biochar application to soils on crop productivity using meta-analysis. Agr. Ecosyst. Environ. 2011, 144, 175-187. [CrossRef]

6. Kookana, R.S.; Sarmah, A.K.; Van Zwieten, L.; Krull, E.; Sing, B. Biochar Application to Soil: Agronomic and Environmental Benefits and Unintended Consequences. In Advances in Agronomy; Sparks, D.L., Ed.; Elsevier Inc: Amsterdam, The Netherlands, 2011; Volume 112, pp. 103-143. [CrossRef]

7. Biederman, L.A.; Harpole, W.S. Biochar and its effects on plant productivity and nutrient cycling: A meta-Analysis. GCB Bioenergy 2013, 5, 202-214. [CrossRef]

8. Mukhergee, A.; Lal, R. Biochar Impacts on Soil Physical Properties and Greenhouse Gas Emissions. Agronomy 2013, 3, 313-339. [CrossRef]

9. Joseph, S.; Camps-Arbestain, M.; Lin, Y.; Munroe, P.; Chia, C.; Hook, J.; Van Zwieten, L.; Kimber, S.; Cowie, A.; Singh, B.; et al. An investigation into the reactions of biochar in soil. Aust. J. Soil Res. 2010, 48, 501-515. [CrossRef]

10. Piccolo, A.; Pietramellara, G.; Mbagwu, J.S.C. Effects of coal derived humic substances on water retention and structural stability of Mediterranean soils. Soil Use Manag. 1996, 12, 209-213. [CrossRef]

11. Laird, D.A. The charcoal vision: A win-win-win scenario for simultaneously producing bioenergy, permanently sequestering carbon, while improving soil and water quality. Agron. J. 2008, 100, 178-181. [CrossRef]

12. Đurđević, B.; Jug, I.; Vukadinović, V.; Brozović, B.; Stipešević, B.; Bogunović, I.; Šeremešić, S.; Jug, D. Effects of Biochar and Sugar Factory Lime Application on Soil Reaction in Acidic Soils. Agric. Conspec. Sci. 2018, 83, 31-37.

13. Mitchell, K.A. The Effect of Biochar on the Growth of Agricultural Weed Species. MSc Thesis, Purdue University, West Lafayette, IN, USA, 3 May 2015; p. 582. Available online: https:/ / docs.lib.purdue.edu/open_access_theses/582 (accessed on 17 July 2021).

14. Volman, J.; Wagentristl, H.; Hartl, W. The effects of simulated weed pressure on early maturity soybeans. Eur. J. Agron. 2010, 32, 243-248. [CrossRef]

15. Travlos, I.S.; Cheimona, N.; Roussis, I.; Bilalis, D.J. Weed-Species Abundance and Diversity Indices in Relation to Tillage Systems and Fertilization. Front. Environ. Sci. 2018, 6, 1-10. [CrossRef]

16. Khan, M.; Haq, N. Wheat crop yield loss assessment due to weeds. Sarhad. J. Agri. 2002, 18, 449-453.

17. Major, J.; Steiner, C.; Ditommaso, A.; Falcão, N.P.S.; Lehmann, J. Weed composition and cover after three years of soil fertility management in the central Brazilian Amazon: Compost, fertilizer, manure and charcoal applications. Weed Biol. Manag. 2005, 5, 69-76. [CrossRef]

18. Quilliam, R.S.; Marsden, K.A.; Gertler, C.; Rousk, J.; DeLuca, T.H.; Jones, D.L. Nutrient dynamics, microbial growth and weed emergence in biochar amended soil are influenced by time since applications and reapplication rate. Agric. Ecosyst. Environ. 2012, 158, 192-199. [CrossRef]

19. Crane-Droesch, A.; Abiven, S.; Jeffery, S.; Torn, M.S. Heterogeneous global crop yield response to biochar: A meta-regression analysis. Environ. Res. Lett. 2013, 8, 1-8. [CrossRef]

20. Balasubramanian, P.; Palaniappan, S.P. Principles and Practices of Agronomy, 2nd ed.; Agrobios: Jodhpur, India, 2004; pp. 306-364.

21. Nie, J.; Yin, L.C.; Liao, Y.L.; Zheng, S.X.; Xie, J. Weed community composition after 26 years of fertilization of late rice. Weed Sci 2009, 57, 256-260. [CrossRef]

22. Cheimona, N.; Angeli, C.; Panagiotou, E.; Tzanidaki, A.; Drontza, C.; Travlos, I.; Bilalis, D. Effect of different types of fertilization on weed flora in processed tomato crop. Agric. Agric. Sci. Procedia 2016, 10, 26-31. [CrossRef]

23. Kaur, S.; Kaur, R.; Chauhan, B.S. Understanding crop-weed-fertilizer-water interactions and their implications for weed management in agricultural systems. Crop. Prot. 2018, 103, 65-72. [CrossRef] 
24. Yin, L.C.; Cai, Z.C.; Zhong, W.H. Changes in weed community diversity of maize crop due to long-term fertilization. Crop. Prot. 2006, 25, 910-914. [CrossRef]

25. Asai, H.; Samson, B.K.; Haefele, M.S.; Songyikhangsuthor, K.; Homma, K.; Kiyono, Y.; Inoue, Y.; Shiraiwa, T.; Horie, T. Biochar amendment techniques for upland rice production in Northern Laos: 1. Soil physical properties, leaf SPAD and grain yield. Field Crops Res. 2009, 111, 81-84. [CrossRef]

26. Steiner, C.; Teixeira, W.G.; Lehmann, J.; Nehls, T.; de Macedo, J.L.V.; Blum, W.E.H.; Zech, W. Long term effects of manure, charcoal and mineral fertilization on crop production and fertility on a highly weathered Central Amazonian upland soil. Plant. Soil 2007, 291, 275-290. [CrossRef]

27. Ali, K.; Arif, M.; Ullah, W.; Abdullah, L.S.; Ahmad, W.; Khan, M.R.; Ayeni, L.S.; Amin, M.; Jehangir, M. Influence of organic and inorganic amendments on weeds density and chemical composition. Pak. J. Weed Sci. Res. 2015, 21, 47-57.

28. Arif, M.; Ali, K.; Haq, M.S.; Khan, Z. Biochar, fym and nitrogen increases weed infestation in wheat. Pak. J. Weed Sci. Res. 2013, 19, 411-418.

29. Abbas, T.; Rizwan, M.; Ali, S.; Adrees, M.; Mahmood, A.; Zia-ur-Rehman, M.; Ibrahim, M.; Arshad, M.; Farooq Qayyum, M. Biochar application increased the growth and yield and reduced cadmium in drought stressed wheat grown in an aged contaminated soil. Ecotoxicol. Environ. Saf. 2018, 148, 825-833. [CrossRef] [PubMed]

30. Bašić, F.; Bogunović, M.; Božić, M.; Husnjak, S.; Jurić, I.; Kisić, I.; Mesić, M.; Mirošević, N.; Romić, D.; Zugec, I. The regionalisation of Croatian agriculture. Agric. Conspec. Sci. 2007, 72, 27-38.

31. WRB. World Reference Base for Soil Resources 2014, Update 2015-International Soil Classification System for Naming Soils and Creating Legends for Soil Maps; World Soil Resources Reports No. 106; FAO: Rome, Italy, 2015.

32. Vukadinović, V.; Vukadinović, V. Plant Nutrition (In Croatian), 3rd ed.; Faculty of Agriculture in Osijek: Osijek, Croatia, 2011; pp. 73-200.

33. International Organization for Standardization ISO 11464. Soil Quality-Pretreatment of Samples for Physico-Chemical Analyses; International Organization for Standardization: Geneva, Switzerland, 2006.

34. International Organization for Standardization ISO 10390. Soil Quality-Determination of pH-An Instrumental Method for the Eoutine Determination of $\mathrm{pH}$; International Organization for Standardization: Geneva, Switzerland, 2005.

35. Egner, H.; Riehm, H.; Domingo, W.R. Untersuchungen über Die Chemische Bodenanalyse als Grundlage für die Beurteilung des Nahrstoffzustandes der Boden, II: Chemische Extractionsmetoden zu Phosphor und Kaliumbestimmung. K. Lantbr. Ann. 1960, $26,199-215$.

36. International Organization for Standardization ISO 14235. Soil Quality—Determination of Organic Carbon by Sulfochromic Oxidation; International Organization for Standardization: Geneva, Switzerland, 2015.

37. Đurđević, B. Practicum in Plant Nutrition (In Croatian), 1st ed.; Faculty of Agriculture in Osijek: Osijek, Croatia, $2014 ;$ pp. 15-17.

38. Tibco Software Inc. Statistica (Data Analysis Software System), Version 13; Tibco Software Inc.: Palo Alto, CA, USA, 2018; Available online: http:/ / tibco.com (accessed on 10 June 2021).

39. Loveland, P.; Webb, J. Is there a critical level of organic matter in the agricultural soils of temperate regions: A review. Soil Till. Res. 2003, 70, 1-18. [CrossRef]

40. Fageria, N.K.; Nascente, A.S. Chapter Six-Management of Soil Acidity of South American Soils for Sustainable Crop Production. In Advances in Agronomy; Sparks, D.L., Ed.; Elsevier Inc.: Amsterdam, The Netherlands, 2014; Volume 128, pp. 221-275.

41. Van Zwieten, L.; Kimbr, S.; Morris, S.; Chan, K.; Downie, A.; Rust, J.; Joseph, S.; Cowie, A. Effects of biochar from slow pyrolysis of papermill waste on agronomic performance and soil fertility. Plant. Soil 2009, 327, 235-246. [CrossRef]

42. Wang, D.; Jiang, P.; Zhang, H.; Yuan, W. Biochar production and applications in agro and forestry systems: A review. Sci. Total Environ. 2020, 723, 137775. [CrossRef]

43. Knežević, M.; Đurkić, M.; Knežević, I.; Antonić, O.; Jelaska, S. Effects of tillage and reduced herbicide doses on weed biomass production in winter and spring cereals. Plant. Soil Environ. 2003, 49, 414-421. [CrossRef]

44. Knežević, M.; Stipešević, B.; Knežević, I.; Lončarić, Z. Weed populations of winter wheat as affected by tillage and nitrogen. Ekológia Bratislava 2007, 26, 190-200.

45. Landolt, E.; Bäumler, B.; Erhardt, A.; Hegg, O.; Klötzli, F.; Lämmler, W.; Nobis, M.; Rudmann-Maurer, K.; Schweingruber, F.H.; Theurillat, J.P.; et al. Ökologische Zeigerwerte und Biologische Kennzeichen zur Flora der Schweiz und der Alpen, 2nd ed.; Landolt, E., Ed.; Haupt Verlag: Bern-Stuttgart-Wien, Switzerland, 2010; pp. 18-20.

46. Radicetti, E.; Mancinelli, R. Sustainable Weed Control in the Agro-Ecosystems. Sustainability 2021, 13, 8639. [CrossRef]

47. Rubinić, V.; Husnjak, S. Clay and Humus Contents Have the Key Impact on Physical Properties of Croatian Pseudogleys. Agric. Conspec. Sci. 2016, 81, 187-191.

48. Patterson, D.T. Weeds in Changing Climate. Weed Sci. 1995, 43, 658-701. [CrossRef]

49. Medlin, C.R.; Shaw, D.R.; Cox, M.S.; Gerard, P.D.; Abshire, M.J.; Wardlaw, M.C. Using soil parameters to predict weed infestation in soybean. Weed Sci. 2001, 49,367-374. [CrossRef]

50. Walter, A.M.; Christensen, S.; Simmelsgaard, S.E. Spatial correlations between weed species densities and soil properties. Weed Res. 2002, 42, 26-38. [CrossRef]

51. Galzina, N.; Barić, K.; Šćepanović, M.; Goršić, M.; Ostojić, Z. Distribution of Invasive Weed Ambrosia artemisiifolia L. in Croatia. Agric. Conspec. Sci. 2010, 75, 75-81. 
52. Abderson, T.N.; Milberg, P. Weed flora and the relative importance of site, crop, crop rotation and nitrogen. Weed. Sci. 1998, 46, 30-38. [CrossRef]

53. Cathcart, R.J.; Swanton, C.J. Nitrogen management will influence threshold values of green foxtail (Setaria viridis) in corn. Weed Sci. 2003, 51, 975-986. [CrossRef]

54. Jurišić, M.; Radočaj, D.; Šiljeg, A.; Antonić, O.; Živić, T. Current status and perspective of remote sensing application in crop management. J. Cent. Eur. Agric. 2021, 22, 156-166. [CrossRef]

55. Arif, M.; Ali, K.; Munsif, F.; Ahmad, W.; Ahmad, A.; Naveed, K. Effects of biochar, fym and nitrogen on weeds and maize phenology. Pak. J. Weed Sci. Res. 2012, 18, 475-484.

56. Smider, B.; Singh, B. Agronomic performance of a high ash biochar in two contrasting soils. Agri. Ecosyst. Environ. 2014, 191, 99-107. [CrossRef]

57. DeLuca, T.H.; Gao, S. Use of biochar in Organic Farming. In Organic Farming; Sarath Chandran, C., Thomas, S., Unn, M., Eds.; Springer: Cham, Switzerland, 2019; pp. 25-49. [CrossRef]

58. Eur-lex.europa.eu. Available online: https:/ / eur-lex.europa.eu/homepage.html (accessed on 14 September 2021).

59. Czekała, W.; Jeżowska, A.; Chełkowski, D. The Use of Biochar for the Production of Organic Fertilizers. J. Ecol. Eng. 2019, 20, 1-8. [CrossRef]

60. Rawat, J.; Saxena, J.; Sanwal, P. Biochar: A Sustainable Approach for Improving Plant Growth and Soil Properties. In Biochar-An Imperative Amendment for Soil and the Environment; Abrol, V., Sharma, P., Eds.; IntechOpen: London, UK, 2019; Available online: https://www.intechopen.com/chapters / 65070 (accessed on 19 August 2021). [CrossRef]

61. Dume, B.; Mosissa, T.; Nebiyu, A. Effect of biochar on soil properties and lead (Pb) availability in a military camp in South West Ethiopia. Afr. J. Environ. Sci. Technol. 2016, 10, 77-85.

62. Schulz, H.; Glaser, B. Effects of biochar compared to organic and inorganic fertilizers on soil quality and plant growth in a greenhouse experiment. J. Plant. Nutr. Soil. Sci. 2012, 175, 410-422. [CrossRef]

63. Alburquerque, J.A.; Salazar, P.; Barrón, V.; Torrent, J.; del Campillo, M.C.; Gallardo, A.; Villar, R. Enhanced wheat yield by biochar addition under different mineral fertilization levels. Agron. Sustain. Dev. 2013, 33, 475-484. [CrossRef] 